\title{
Non-ergodicity of Nosé-Hoover dynamics
}

\author{
Frédéric Legoll ${ }^{1,2}$, Mitchell Luskin ${ }^{3}$ and Richard Moeckel ${ }^{3}$ \\ ${ }^{1}$ Université Paris-Est, Institut Navier, LAMI, École Nationale des Ponts et \\ Chaussées, 6 et 8 avenue Blaise Pascal, 77455 Marne-La-Vallée Cedex 2, France \\ ${ }^{2}$ INRIA Rocquencourt, MICMAC Team-Project, Domaine de Voluceau, B.P. 105, \\ 78153 Le Chesnay Cedex, France \\ ${ }^{3}$ School of Mathematics, University of Minnesota, 206 Church Street SE, \\ Minneapolis, MN 55455, U.S.A.
}

E-mail: legoll@lami.enpc.fr, luskin@math.umn.edu, rick@math.umn.edu

\begin{abstract}
The numerical integration of the Nosé-Hoover dynamics gives a deterministic method that is used to sample the canonical Gibbs measure. The Nosé-Hoover dynamics extends the physical Hamiltonian dynamics by the addition of a "thermostat" variable, that is coupled nonlinearly with the physical variables. The accuracy of the method depends on the dynamics being ergodic. Numerical experiments have been published earlier that are consistent with non-ergodicity of the dynamics for some model problems. The authors recently proved the non-ergodicity of the Nosé-Hoover dynamics for the one-dimensional harmonic oscillator.

In this paper, this result is extended to non-harmonic one-dimensional systems. It is also shown for some multidimensional systems that the averaged dynamics for the limit of infinite thermostat "mass" have many invariants, thus giving theoretical support for either non-ergodicity or slow ergodization. Numerical experiments for a two-dimensional central force problem and the one-dimensional pendulum problem give evidence for non-ergodicity.
\end{abstract}

AMS classification scheme numbers: 37M25, 65P10, 70F10, 82B80 


\section{Introduction}

The computation of equilibrium statistical properties of molecular systems is of great importance in materials science, computational physics, chemistry, and biology [6, 14]. These equilibrium statistical properties are given by phase space integrals of the form

$$
\langle A\rangle=\int A(q, p) d \mu(q, p)
$$

where $q=\left(q_{1}, \ldots, q_{N}\right) \in \mathbb{R}^{N}$ and $p=\left(p_{1}, \ldots, p_{N}\right) \in \mathbb{R}^{N}$ denote a set of positions and momenta and $A(q, p)$ is an observable, a function defined over the phase space and related to the macroscopic quantity under study. The computation of integrals such as (1) is often a challenging problem, especially when the number of degrees of freedom is large.

For molecular systems at fixed temperature $\theta$, the measure $d \mu$ is the Gibbs measure for the canonical ensemble [6, 14]

$$
d \mu(q, p)=\left[\frac{\exp (-\beta H(q, p))}{\int \exp (-\beta H(q, p)) d q d p}\right] d q d p,
$$

where $H(q, p)$ is the Hamiltonian of the system and $\beta$ is related to the temperature $\theta$ by $\beta=1 /\left(k_{B} \theta\right)$ with $k_{B}$ denoting the Boltzmann constant. We will consider Hamiltonians of the general form

$$
H(q, p)=\frac{p^{T} M^{-1}(q) p}{2}+V(q)
$$

where $M(q) \in \mathbb{R}^{N \times N}$ for $q \in \mathbb{R}^{N}$ is the generalized mass matrix and $V(q)$ is the potential energy. We assume that the generalized mass matrix $M(q) \in \mathbb{R}^{N \times N}$ is symmetric and positive definite, so its inverse $M^{-1}(q) \in \mathbb{R}^{N \times N}$ exists for all $q \in \mathbb{R}^{N}$ and is also symmetric and positive definite.

Many methods have been proposed and utilized to approximate the phase space integral (11), including methods based on stochastic or deterministic dynamics for $(q, p)$. If the dynamics is ergodic with respect to the measure $d \mu$ given by (2), then the phasespace average (1) is equal to the time average

$$
\int A(q, p) d \mu(q, p)=\lim _{T \rightarrow+\infty} \frac{1}{T} \int_{0}^{T} A(q(t), p(t)) d t
$$

over a trajectory $(q(t), p(t))_{t \geq 0}$. Thus, the time average can be approximated by

$$
\lim _{T \rightarrow+\infty} \frac{1}{T} \int_{0}^{T} A(q(t), p(t)) d t \approx \lim _{\mathcal{N} \rightarrow+\infty} \frac{1}{\mathcal{N}} \sum_{\ell=1}^{\mathcal{N}} A\left(q_{\ell}, p_{\ell}\right)
$$

where $\left(q_{\ell}, p_{\ell}\right)_{\ell \geq 1}$ is a numerical solution of the chosen dynamics.

In this paper, we investigate the deterministic dynamics known as Nosé-Hoover dynamics [7, which is still widely used although variants have been developed with the goal to improve its efficiency and overcome its deficiencies [12, 16, 3, 11]. This dynamics 
has been first proposed in the form of a Hamiltonian dynamics on an extended phase space [15], the Hamiltonian being chosen such that the marginal distribution of its microcanonical density is the canonical Gibbs density for the physical variables. The Nosé-Hoover dynamics is then constructed by rescaling time and momentum to obtain a non-Hamiltonian dynamics with physical time and momentum [7].

Stochastic dynamics (such as the Langevin equation, or the recently proposed Hoover-Langevin method [10]) can also be considered. See [4] for a review of sampling methods of the canonical ensemble, along with a theoretical and numerical comparison of their performances for molecular dynamics.

The equality (4) relies on an ergodicity condition. This condition has been rigorously proven neither for the Nosé-Hoover dynamics, nor for any other deterministic method commonly used in practice. In fact, there is numerical evidence that shows that the Nosé-Hoover method is not ergodic for some systems [7, 12, 16], including the onedimensional harmonic oscillator. In [9], we have rigorously analyzed the dynamics in this special case, and indeed proven the non-ergodicity, for some regime of parameters.

In this article, we study more general systems. After briefly recalling the NoséHoover equations (see Section 2), we first consider a class of multidimensional systems (see Section 3). Taking the limit of an infinite thermostat "mass" in the Nosé-Hoover equations, we formally obtain an averaged dynamics, for which we prove the existence of many invariants. These theoretical results are illustrated by numerical simulations of a specific system (see Section 4). We numerically observe that, for finite thermostat mass, these invariants are of course not exactly preserved, but still remain close to their initial value. This prevents the Nosé-Hoover system from thermalizing. In Section 5, we next turn to the one-dimensional case, for which we obtain stronger results. We first prove non-ergodicity of the Nosé-Hoover dynamics, when the mass of the thermostat is large enough (see Section 5.1). Our method extends the one we used to study the harmonic oscillator case [9]. Section 5.2 describes an example of such a one degree of freedom problem. Again, numerical simulations illustrate the obtained theoretical results.

\section{Nosé-Hoover dynamics}

The Nosé-Hoover dynamics involves the physical variables $q$ and $p$ and one additional scalar variable, $\xi$, which represents the momentum of a thermal bath exchanging energy with the system. The differential equations are:

$$
\begin{aligned}
\dot{q} & =\frac{\partial H}{\partial p}=M^{-1}(q) p \\
\dot{p} & =-\frac{\partial H}{\partial q}-\frac{\xi}{Q} p=-\nabla V(q)-\frac{p^{T} \nabla M^{-1}(q) p}{2}-\frac{\xi}{Q} p \\
\dot{\xi} & =p^{T} M^{-1}(q) p-\frac{N}{\beta}
\end{aligned}
$$

where - denotes the time-derivative. The parameter $Q$ represents the mass of the thermostat; it is a free parameter that the user has to choose. 
We recall that invariant measures $\rho(z) d z$ for a general dynamical system

$$
\dot{z}=f(z)
$$

are determined by the equilibrium equation

$$
\operatorname{div}(\rho(z) f(z))=0 .
$$

It can be verified by direct computation that the dynamics (5) preserves the measure

$$
d \mu_{\mathrm{NH}}=\exp \left[-\beta\left(H(q, p)+\frac{\xi^{2}}{2 Q}\right)\right] d q d p d \xi
$$

by using the fact that the kinetic energy $\frac{p^{T} M^{-1}(q) p}{2}$ is quadratic in $p$.

If the dynamics (5) is ergodic with respect to $d \mu_{\mathrm{NH}}$, then, by integrating out $\xi$, we have that the dynamics $(q(t), p(t))$ is ergodic with respect to the Gibbs measure. In this case, the time-average of a function $A(q, p)$ along a typical Nosé-Hoover trajectory provides an estimate for the space-average of $A$ with respect to Gibbs measure. Unfortunately, the system is generally not ergodic. In [9] we proved non-ergodicity in the case of the one-dimensional harmonic oscillator. Our aim here is to study more general systems.

\section{Systems with first integrals}

In this section, we show how the presence of additional integrals for a Hamiltonian system can impede ergodization of the Nosé-Hoover dynamics.

\subsection{Homogeneous integrals}

Consider a Hamiltonian system

$$
\dot{q}=\frac{\partial H}{\partial p}, \quad \dot{p}=-\frac{\partial H}{\partial q},
$$

for energy (3) which admits a first integral other than $H$ itself. This means that there is a smooth function $F(q, p)$ whose Poisson bracket with $H$ vanishes, i.e.,

$$
\{H, F\}=H_{q}^{T} F_{p}-H_{p}^{T} F_{q}=0 .
$$

If $F$ is a homogeneous function of the momentum variables, then it gives rise to a first integral of the Nosé-Hoover system.

Theorem 3.1 If $F(q, p)$ is a first integral of (3) which is homogeneous of degree $k$ with respect to the momentum variables, $p$, then

$$
G(q, p, \xi)=\frac{\xi^{2}}{2 Q}+H(q, p)-\frac{N}{\beta k} \ln |F(q, p)|
$$

is a first integral of the corresponding Nosé-Hoover system (5).

The proof is a simple computation using (8), (5) and the fact that $F_{p}^{T} p=k F$.

Of course, the existence of such an integral immediately gives non-ergodicity of the Nosé-Hoover system with respect to (6) . For a simple example of a system admitting such a homogeneous integral, see Section 4 . 


\subsection{Completely integrable systems and action-angle variables}

We now assume that the Hamiltonian dynamical system (7) is completely integrable, i.e., the system admits $N$ independent first integrals which commute in the sense that the Poisson brackets of any two of them vanish [1]. The rest of this section is devoted to showing that these integrals, even if they are not homogeneous, have a deleterious effect on the ergodization of the corresponding Nosé-Hoover system.

The non-degenerate level sets of the integrals are $N$-dimensional manifolds and if they are compact then their connected components are diffeomorphic to the $N$ dimensional torus $\mathbb{T}^{N}$. Moreover, such a torus has a neighborhood $U \subset \mathbb{R}^{N} \times \mathbb{R}^{N}$ in which one can introduce symplectic action-angle variables. More precisely, there exist angle variables $\theta \in \mathbb{T}^{N}$, action variables $a \in D \subset \mathbb{R}^{N}$, and a symplectic diffeomorphism $\psi: U \rightarrow \mathbb{T}^{N} \times D$ which transforms (7) to the form

$$
\dot{\theta}=\omega(a), \quad \dot{a}=0 .
$$

Here $D$ is an open subset of $\mathbb{R}^{N}$. Equivalently, the action-angle Hamiltonian $\tilde{H}(\theta, a)=$ $H\left(\psi^{-1}(\theta, a)\right)$ is independent of $\theta$ and $\frac{\partial \tilde{H}(\theta, a)}{\partial a}=\omega(a)$.

In what follows, it will be convenient to define the angle mapping $\psi_{1}(q, p) \in \mathbb{R}^{N}$ and the action mapping $\psi_{2}(q, p) \in \mathbb{R}^{N}$ by $\psi(q, p)=\left(\psi_{1}(q, p), \psi_{2}(q, p)\right) \in \mathbb{R}^{2 N}$. We will also use the abbreviated notation $\partial_{1} \psi_{i}=\frac{\partial \psi_{i}}{\partial q} \in \mathbb{R}^{N \times N}$ and $\partial_{2} \psi_{i}=\frac{\partial \psi_{i}}{\partial p} \in \mathbb{R}^{N \times N}$. We then denote the Jacobian of $\psi$ by

$$
\mathrm{D} \psi=\left(\begin{array}{cc}
\partial_{1} \psi_{1} & \partial_{2} \psi_{1} \\
\partial_{1} \psi_{2} & \partial_{2} \psi_{2}
\end{array}\right)
$$

We denote the inverse mapping of $\psi(q, p)$ by $\phi(\theta, a)$. The matrix $(\mathrm{D} \psi)=(\mathrm{D} \phi)^{-1}$ has a simple form since $\phi$ is symplectic:

$$
(\mathrm{D} \psi)=J^{-1}(\mathrm{D} \phi)^{T} J=\left(\begin{array}{cc}
\left(\partial_{2} \phi_{2}\right)^{T} & -\left(\partial_{2} \phi_{1}\right)^{T} \\
-\left(\partial_{1} \phi_{2}\right)^{T} & \left(\partial_{1} \phi_{1}\right)^{T}
\end{array}\right),
$$

where

$$
J=\left(\begin{array}{rc}
0 & I_{N} \\
-I_{N} & 0
\end{array}\right)
$$

The diffeomorphism $\psi$ transforms (7) to (10) by the chain rule

$$
\left(\begin{array}{c}
\dot{\theta} \\
\dot{a}
\end{array}\right)=\mathrm{D} \psi\left(\begin{array}{c}
\dot{q} \\
\dot{p}
\end{array}\right) \text {. }
$$

Since $\psi$ is symplectic, the dynamics (10) is obtained from the Hamiltonian $\tilde{H}(\theta, a)$. Hence,

$$
\left(\begin{array}{c}
\omega(a) \\
0
\end{array}\right)=\left(\begin{array}{c}
\frac{\partial \tilde{H}}{\partial a} \\
-\frac{\partial \tilde{H}}{\partial \theta}
\end{array}\right)=\mathrm{D} \psi\left(\begin{array}{c}
\frac{\partial H}{\partial p} \\
-\frac{\partial H}{\partial q}
\end{array}\right)
$$




\subsection{Recasting the Nosé-Hoover dynamics}

We now multiply the Nosé-Hoover equations (5) for $\dot{q}$ and $\dot{p}$ by $\mathrm{D} \psi$ to obtain from (12) and (13) that

$$
\left(\begin{array}{l}
\dot{\theta} \\
\dot{a}
\end{array}\right)=\left(\begin{array}{c}
\omega(a) \\
0
\end{array}\right)-\frac{\xi}{Q} \mathrm{D} \psi\left(\begin{array}{l}
0 \\
p
\end{array}\right) .
$$

As we are interested in the regime $Q \gg 1$, we rescale by

$$
\varepsilon=1 / \sqrt{Q}, \quad \alpha=\xi / \sqrt{Q} .
$$

Using the symplectic property (11) of $\psi$, we see that the Nosé-Hoover equation (5) can be then given in the scaled angle-action variables by

$$
\left(\begin{array}{c}
\dot{\theta} \\
\dot{a} \\
\dot{\alpha}
\end{array}\right)=\left(\begin{array}{c}
\omega(a) \\
0 \\
0
\end{array}\right)+\varepsilon\left(\begin{array}{c}
\alpha\left(\partial_{2} \phi_{1}\right)^{T} \phi_{2} \\
-\alpha\left(\partial_{1} \phi_{1}\right)^{T} \phi_{2} \\
\phi_{2}^{T} M^{-1}\left(\phi_{1}\right) \phi_{2}-N \beta^{-1}
\end{array}\right) .
$$

\subsection{Averaging the fast variables}

We next apply the averaging method to obtain an approximate system which does not involve the fast variables. Rigorous results about averaging for Hamiltonian systems with several degrees of freedom are fraught with technical difficulties (see for example [2]). These arise from the fact that $\omega(a)$, the frequency vector of the fast angles, experiences resonances of the form $k \cdot \omega(a)=0, k \in \mathbb{Z}^{N}$, for certain values of the action vector $a$. In fact, these resonant actions are generally dense in the action domain $D$. In spite of this, the averaged differential equations often provide a useful first approximation to the behavior of the slow variables when $\varepsilon$ is small.

In our problem, the averaged system for the slow variables is given by

$$
\begin{aligned}
\dot{a} & =-\alpha S(a), \\
\dot{\alpha} & =k(a),
\end{aligned}
$$

where

$$
\begin{aligned}
S(a) & =\left\langle\left(\partial_{1} \phi_{1}\right)^{T} \phi_{2}\right\rangle(a)=\int_{\mathbb{T}^{N}}\left(\partial_{1} \phi_{1}\right)^{T}(\theta, a) \phi_{2}(\theta, a) d \theta, \\
k(a) & =\left\langle\phi_{2}^{T} M^{-1}\left(\phi_{1}\right) \phi_{2}\right\rangle(a)-\frac{N}{\beta} \\
& =\int_{\mathbb{T}^{N}} \phi_{2}^{T}(\theta, a) M^{-1}\left(\phi_{1}(\theta, a)\right) \phi_{2}(\theta, a) d \theta-\frac{N}{\beta} .
\end{aligned}
$$

We next show that, with an additional assumption on the action-angle mapping $\phi$, we have

$$
S(a)=a .
$$

Recall that a map $(q, p)=\phi(\theta, a)$ is symplectic if it preserves the canonical differential two-forms, i.e., $\phi^{*}\left(\sum d p_{i} \wedge d q_{i}\right)=\sum d a_{i} \wedge d \theta_{i}$ where $\phi^{*}$ indicates the pull-back, meaning that we write $p_{i}, q_{i}, d p_{i}, d q_{i}$ in terms of the $\theta, a$ variables. It follows from this that the 
difference of the corresponding canonical one-forms $\phi^{*}\left(p^{T} d q\right)-a^{T} d \theta$ is a closed oneform on $\mathbb{T}^{N} \times D$. We will call $\phi$ exact symplectic if this closed one-form is exact, i.e., if

$$
\phi^{*}\left(p^{T} d q\right)=a^{T} d \theta+d F(\theta, a)
$$

where $F(\theta, a)$ is a real-valued function on $\mathbb{T}^{N} \times D$. This stronger condition holds for the action-angle coordinates associated to many well-known integrable systems.

As an example, consider the one-dimensional harmonic oscillator $H(q, p)=\frac{1}{2}\left(p^{2}+\right.$ $q^{2}$ ) which is a completely integrable system with $N=1$ degrees of freedom. In any annulus of the form $0<h_{1} \leq\left(p^{2}+q^{2}\right) / 2 \leq h_{2}$, we can introduce action-angle variables $(\theta, a)$ such that

$$
q=\sqrt{2 a} \cos \theta, \quad p=-\sqrt{2 a} \sin \theta .
$$

For $\phi(\theta, a)=(q, p)$, we have

$$
\phi^{*}(p d q)=-\sin \theta \cos \theta d a+2 a \sin ^{2} \theta d \theta
$$

and so

$$
\phi^{*}(p d q)-a d \theta=-\sin \theta \cos \theta d a+a\left(2 \sin ^{2} \theta-1\right) d \theta=d F
$$

where

$$
F=-a \sin \theta \cos \theta
$$

It turns out that the action-angle variables constructed according to the usual method of Arnold [1] always have this exactness property. To see this, recall that in Arnold's method, the tori given by fixing the $N$-independent integrals of motion are parametrized by angle variables $\theta=\left(\theta_{1}, \ldots, \theta_{N}\right)$ derived from the $N$ commuting Hamiltonian flows defined by the integrals. Then the action variables are given by

$$
a_{i}=\int_{\gamma_{i}} p^{T} d q
$$

where $\gamma_{i}$ is the curve in the torus defined by holding $\theta_{j}=$ const for $j \neq i$ and letting $\theta_{i}$ run over $[0,1]$. The integral depends on which torus is considered, i.e., it is a function of the $N$ first integrals. The usual proof shows that the map $(q, p)=\phi(\theta, a)$ is defined and symplectic on some domain of the form $\mathbb{T}^{N} \times D$. It follows that

$$
\nu=\phi^{*}\left(p^{T} d q\right)-a d \theta
$$

is a closed differential one-form on $\mathbb{T}^{N} \times D$. Showing that $\phi$ is exact symplectic amounts to showing that $\nu$ is exact. For this, it suffices to check that its integral around any closed curve vanishes. In fact, since $\nu$ is closed, it suffices to check the curves of the form $C_{i}=\Gamma_{i} \times\left\{a_{0}\right\}, a_{0} \in D$ where $\Gamma_{i}=\left\{\theta: \theta_{j}=\right.$ const, $\left.j \neq i\right\}$. For such a curve, we have

$$
\int_{C_{i}} \nu=\int_{C_{i}} \phi^{*}(p d q)-a d \theta=\int_{\gamma_{i}} p d q-\int_{0}^{1} a_{0 i} d \theta_{i}=a_{0 i}-a_{0 i}=0 .
$$

Here we used the fact that under $\phi$ the curve $C_{i}$ maps to the curve $\gamma_{i}$ used in (19). 
To prove (17) under the exact symplectic assumption (18), we first note that

$$
\phi^{*}\left(p^{T} d q\right)=\phi_{2}^{T} d \phi_{1}=\phi_{2}^{T}\left(\partial_{1} \phi_{1} d \theta+\partial_{2} \phi_{1} d a\right) .
$$

Hence, (18) reads

$$
\phi_{2}^{T}\left(\partial_{1} \phi_{1} d \theta+\partial_{2} \phi_{1} d a\right)=a^{T} d \theta+d F(\theta, a) .
$$

For any $j=1, \ldots, N$, we can integrate both sides with respect to $\theta_{j}$, along the circular loop $C_{j}$ as in the last paragraph. Using the periodicity of $F$ in $\theta_{j}$, we obtain

$$
\sum_{i=1}^{N} \int_{0}^{1}\left(\frac{\partial \phi_{1 i}}{\partial \theta_{j}}\right)(\theta, a) \phi_{2 i}(\theta, a) d \theta_{j}=a_{j} .
$$

We can then further integrate (20) over the angles $\theta_{k}$ for $k \neq j$ to obtain that

$$
S(a)_{j}=\sum_{i=1}^{N} \int_{\mathbb{T}^{N}}\left(\frac{\partial \phi_{1 i}}{\partial \theta_{j}}\right)(\theta, a) \phi_{2 i}(\theta, a) d \theta=a_{j}
$$

for $j=1, \ldots, N$.

\subsection{First integrals of the averaged Nosé-Hoover equations}

A direct calculation shows that a set of $N$ independent first integrals for the averaged Nosé-Hoover equations

$$
\begin{aligned}
\dot{a} & =-\alpha a, \\
\dot{\alpha} & =k(a),
\end{aligned}
$$

are given by

$$
\begin{aligned}
G_{i}(a, \alpha) & =\frac{a_{i}}{a_{N}}, \quad i=1, \ldots, N-1 \\
G_{N}(a, \alpha) & =\frac{\alpha^{2}}{2}+\int \frac{k\left(s \frac{a_{1}}{a_{N}}, \ldots, s \frac{a_{N-1}}{a_{N}}, s\right)}{s} d s .
\end{aligned}
$$

To prove that $G_{N}(a, \alpha)$ is a first integral, it is helpful to use the fact that $G_{i}(a, \alpha)=$ $a_{i} / a_{N}$ for $i=1, \ldots, N-1$ is a first integral.

We summarize the result of this section in the following theorem.

Theorem 3.2 The averaged equations for the Nosé-Hoover dynamics for a completely integrable Hamiltonian system has $N$ independent first integrals.

To the extent that the averaging method applies, we expect that $G_{i}(a(t), \alpha(t))$ evolves slowly for small $\varepsilon$ and so the sampling of the Gibbs measure is slow even if the dynamics is ergodic. We will verify this numerically in an example in the next section. It turns out that $G_{i}(a(t), \alpha(t))$ remains quite close to its initial value for fairly large values of $\varepsilon$ as well. 


\section{A central force problem}

We consider here a two degrees of freedom system to illustrate the theoretical results obtained in the previous section. We work with the Hamiltonian (3) with $N=2$, the identity mass matrix $M(q)=I_{2}$, and a potential $V(|q|)$ which depends only on the distance to the origin. The Hamiltonian system (7) admits two first integrals, the energy $H$ and the angular momentum

$$
L=q_{1} p_{2}-q_{2} p_{1},
$$

which satisfy $\{H, L\}=0$, and whose gradients are linearly independent, except for values of $H$ and $L$ satisfying a condition of the form $f(H, L)=0$ for some function $f$. Hence, this system is completely integrable. Assume that $V(r) \rightarrow+\infty$ as $r \rightarrow+\infty$. Then level sets of $H$ are compact, hence the level sets $\left\{(q, p) \in \mathbb{R}^{4} ; H(q, p)=h, L(q, p)=\ell\right\}$ are also compact, hence there exists action-angle variables for this system.

To describe the action variables, first introduce polar coordinates $(r, \phi)$ in $\mathbb{R}^{2}$. The angular momentum is

$$
L=r^{2} \dot{\phi}
$$

Fixing a value for $L$, we have a reduced Hamiltonian system for the radial variables $\left(r, p_{r}\right)$, where $p_{r}=\dot{r}$, with Hamiltonian

$$
H_{L}\left(r, p_{r}\right)=\frac{1}{2} p_{r}^{2}+\frac{1}{2} \frac{L^{2}}{r^{2}}+V(r)
$$

This reduced system has one degree of freedom and can be understood by the usual phase-plane method. Since $V(r) \rightarrow+\infty$ as $r \rightarrow+\infty$, the level curves

$$
C_{(h, L)}=\left\{\left(r, p_{r}\right): H_{L}\left(r, p_{r}\right)=h\right\}, \quad L \neq 0,
$$

generically consist of one or more simple closed curves. For the unreduced system, where we remember the angle $\phi$, each such curve becomes an invariant torus $T_{(h, L)}$. It can be shown that the action variables assigned to such a torus by Arnold's procedure are as follows: $a_{1}(h, L)$ is the area in the $\left(r, p_{r}\right)$ plane enclosed by the simple closed curve of the reduced system,

$$
a_{1}(h, L)=\int_{C_{(h, L)}} p_{r} d r
$$

and $a_{2}(h, L)=L$, the angular momentum. Note that $a_{1}(h, L)$ is easily computable by standard numerical integration schemes.

Since $L$ is homogeneous of degree $k=1$ in the momentum variables, Theorem 3.1 gives a first integral for the Nosé-Hoover system:

$$
G(q, p, \xi)=\frac{\xi^{2}}{2 Q}+H(q, p)-\frac{2}{\beta} \ln |L(q, p)| .
$$

In addition, Theorem 3.2 provides additional integrals for the averaged Nosé-Hoover equations, in particular the ratio of the action variables

$$
G_{1}(q, p)=\frac{a_{1}(q, p)}{a_{2}(q, p)}=\frac{a_{1}(H(q, p), L(q, p))}{L(q, p)} .
$$


To the extent that the averaging method applies for this two-degrees of freedom problem, this ratio should evolve only very slowly when $Q \gg 1$. In the sequel, we present some numerical simulations showing first that, when $Q \gg 1$, this is indeed the case, and second that, for $Q=1$, such a behaviour persists to some extent.

Consider the example with potential

$$
V(r)=r^{2}+r^{4}
$$

with an initial condition $\left(q_{0}, p_{0}, \xi_{0}\right)$ such that $L\left(q_{0}, p_{0}\right) \neq 0$. We compute the trajectory of the Nosé-Hoover dynamics (51) with the algorithm proposed in [13]. On Figure 1, we plot $G(q(t), p(t), \xi(t))$ and $G_{1}(q(t), p(t))$, where $G$ and $G_{1}$ are defined by (23) and (24), for $Q=100$. We indeed observe that $G$ is preserved, whereas $G_{1}$ evolves slowly.

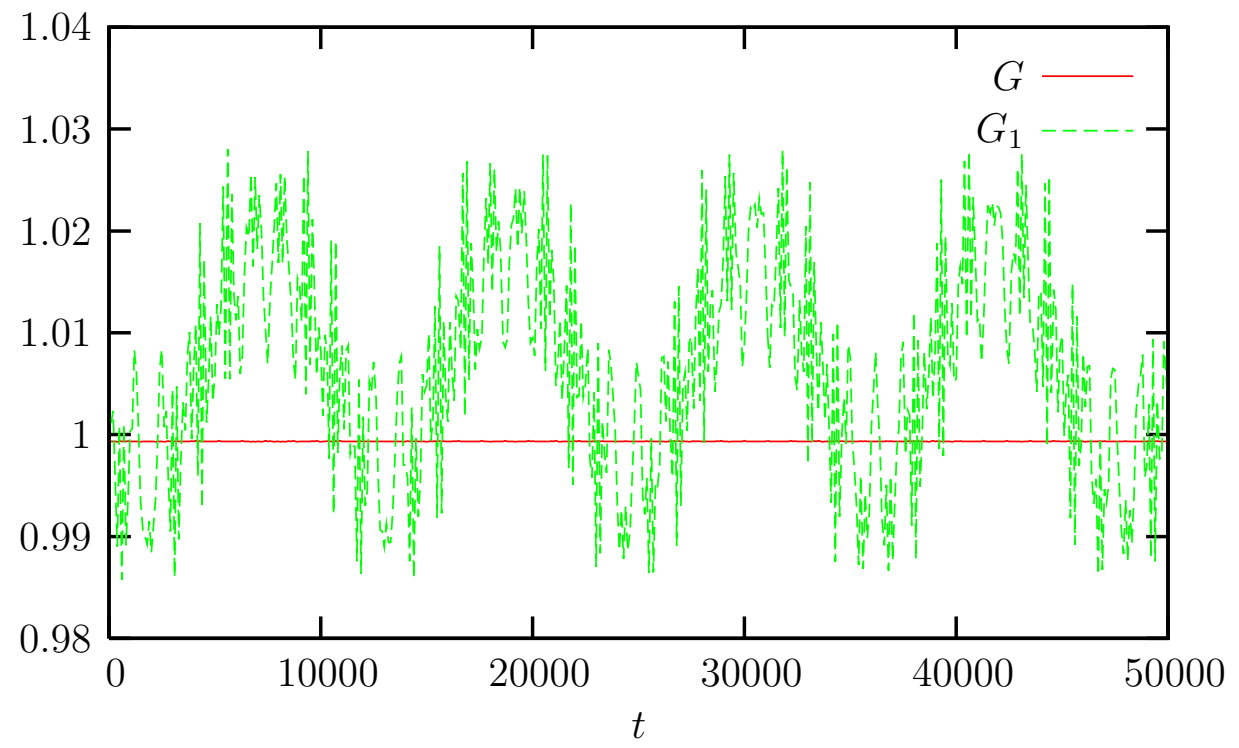

Figure 1. Plot of $G(q(t), p(t), \xi(t))$ and $G_{1}(q(t), p(t))$ (renormalized by their initial value) along the trajectory of (5), for $Q=100(\beta=1$, initial condition $q=(0 ; 0.5)$, $p=(-1.5 ; 1.5), \xi=0)$.

We now consider the value $Q=1$ and plot the same quantities as above on Figure 2 . Again, $G$ is preserved, whereas $G_{1}$ evolves in a band which is still quite narrow, even for this small value of $Q$.

Let us now derive another quantity, which does not behave as well as $G_{1}$ for large $Q$, but happens to behave in a better way for small $Q \dagger$. From the Nosé-Hoover dynamics (5), we compute that

$$
\begin{aligned}
\dot{L} & =-\frac{\xi}{Q} L=-\varepsilon \alpha L, \\
\dot{H} & =-\frac{\xi}{Q} p^{T} p=-\varepsilon \alpha p^{T} p=-\varepsilon \alpha \phi_{2}^{T}(\theta, a) \phi_{2}(\theta, a) .
\end{aligned}
$$

$\ddagger$ We have a clear understanding of why $G_{1}$ behaves better than this quantity when $Q \gg 1$. However, the situation for $Q=1$ is less clear. 




Figure 2. Plot of $G(q(t), p(t), \xi(t))$ and $G_{1}(q(t), p(t))$ (renormalized by their initial value) along the trajectory of (5), for $Q=1(\beta=1$, initial condition $q=(0 ; 0.5)$, $p=(-1.5 ; 1.5), \xi=0)$.

Since $\theta$ are fast variables whereas $a, \alpha$ and $H$ are slow ones, we again formally use the averaging method on (26) and consider the dynamics

$$
\dot{H}=-\varepsilon \alpha k_{0}(a)
$$

with

$$
k_{0}(a)=\int_{\mathbb{T}^{2}} \phi_{2}^{T}(\theta, a) \phi_{2}(\theta, a) d \theta
$$

(note that (25) does not depend on the fast variables $\theta$ ). Now recall that the action variables $a$ are functions of $H$ and $L$. The equation (27) hence reads

$$
\dot{H}=-\varepsilon \alpha k_{0}(H, L) \text {. }
$$

The averaged system is thus

$$
\begin{aligned}
\dot{L} & =-\alpha L, \\
\dot{H} & =-\alpha k_{0}(H, L), \\
\dot{\alpha} & =k_{0}(H, L)-2 \beta^{-1} .
\end{aligned}
$$

Note that

$$
E(H, L, \alpha)=\frac{\alpha^{2}}{2}+H-\frac{2}{\beta} \ln |L|
$$

is a first integral of the above system. It is just the analogue of (23) being a first integral for the Nosé-Hoover system (see Theorem 3.1).

On Figure 3, we plot the function $H \mapsto k_{0}(H, L)$, for several values of $L$. We observe that $k_{0}(H, L)$ is almost a constant with respect to $L$, and can hence be approximated $\S$ by a function $k_{0}^{\mathrm{app}}(H)$.

$\S$ In practice, we have considered several energy values $H_{i}$, and for each $H_{i}$, we have considered several configurations $\left(q_{i, j}, p_{i, j}\right)$ with energy $H_{i}$ and angular momentum $L_{i, j}$. We next have computed 


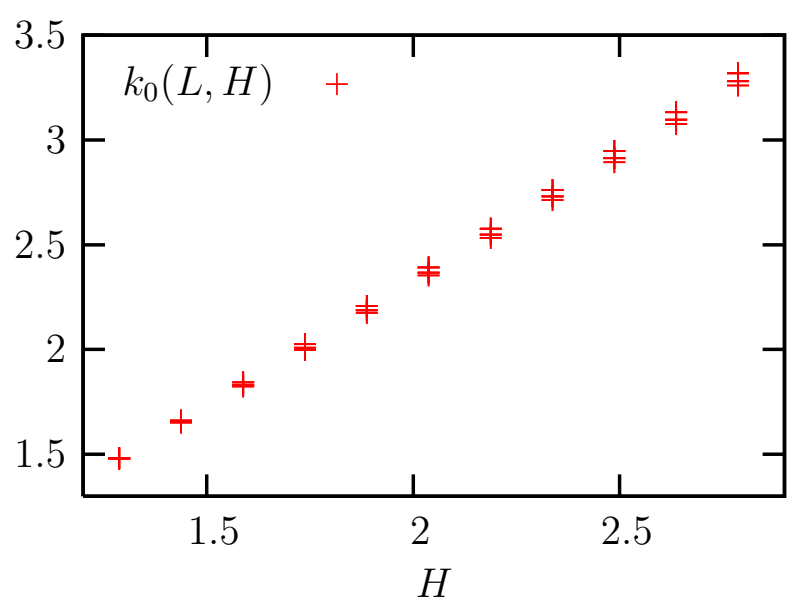

Figure 3. Plot of $H \mapsto k_{0}(L, H)$. For each value of $H$, we have considered several values of $L$.

We hence approximate (28) by

$$
\begin{aligned}
\dot{L} & =-\alpha L, \\
\dot{H} & =-\alpha k_{0}^{\mathrm{app}}(H), \\
\dot{\alpha} & =k_{0}^{\mathrm{app}}(H)-2 \beta^{-1} .
\end{aligned}
$$

Now, it is natural to introduce the variable $\tau$ defined by

$$
\tau(H)=\exp \left(\int^{H} \frac{d s}{k_{0}^{\mathrm{app}}(s)}\right)
$$

and its reciprocal $H(\tau)$, such that (30) reads

$$
\begin{aligned}
\dot{L} & =-\alpha L, \\
\dot{\tau} & =-\alpha \tau, \\
\dot{\alpha} & =k_{0}^{\mathrm{app}}(H(\tau))-2 \beta^{-1} .
\end{aligned}
$$

This system is in the form (21). Its two first integrals are

$$
E_{1}(L, \tau)=\frac{\tau}{L}
$$

and

$$
\begin{aligned}
E_{2}(\tau, \alpha) & =\frac{\alpha^{2}}{2}+\int^{\tau} \frac{k_{0}^{\mathrm{app}}(H(s))-2 \beta^{-1}}{s} d s \\
& =\frac{\alpha^{2}}{2}+\int^{\tau} \frac{k_{0}^{\mathrm{app}}(H(s))}{s} d s-\frac{2}{\beta} \ln \tau \\
& =\frac{\alpha^{2}}{2}+H(\tau)-\frac{2}{\beta} \ln \tau .
\end{aligned}
$$

The first invariant $E$ given by (29) is not independent from $E_{1}$ and $E_{2}$ : $E_{2}=$ $E-2 \beta^{-1} \ln \left|E_{1}\right|$.

$k_{0}\left(H_{i}, L_{i, j}\right)$ by averaging $p(t)^{T} p(t)$ along a constant energy trajectory. Averaging these $k_{0}\left(H_{i}, L_{i, j}\right)$, we obtain $k_{0}^{\text {app }}\left(H_{i}\right)$, which next leads to $k_{0}^{\text {app }}(H)$ for any $H$ by piecewise linear interpolation. 
We now consider the same trajectories of the Nosé-Hoover dynamics that we considered on Figures 1 and 2, and we plot

$$
E_{1}(q, p)=E_{1}(L(q, p), \tau(H(q, p))) .
$$

We see on Figure 4 that, for $Q=100$, this quantity is almost preserved, and that, even for $Q=1$, it remains close to its initial value.

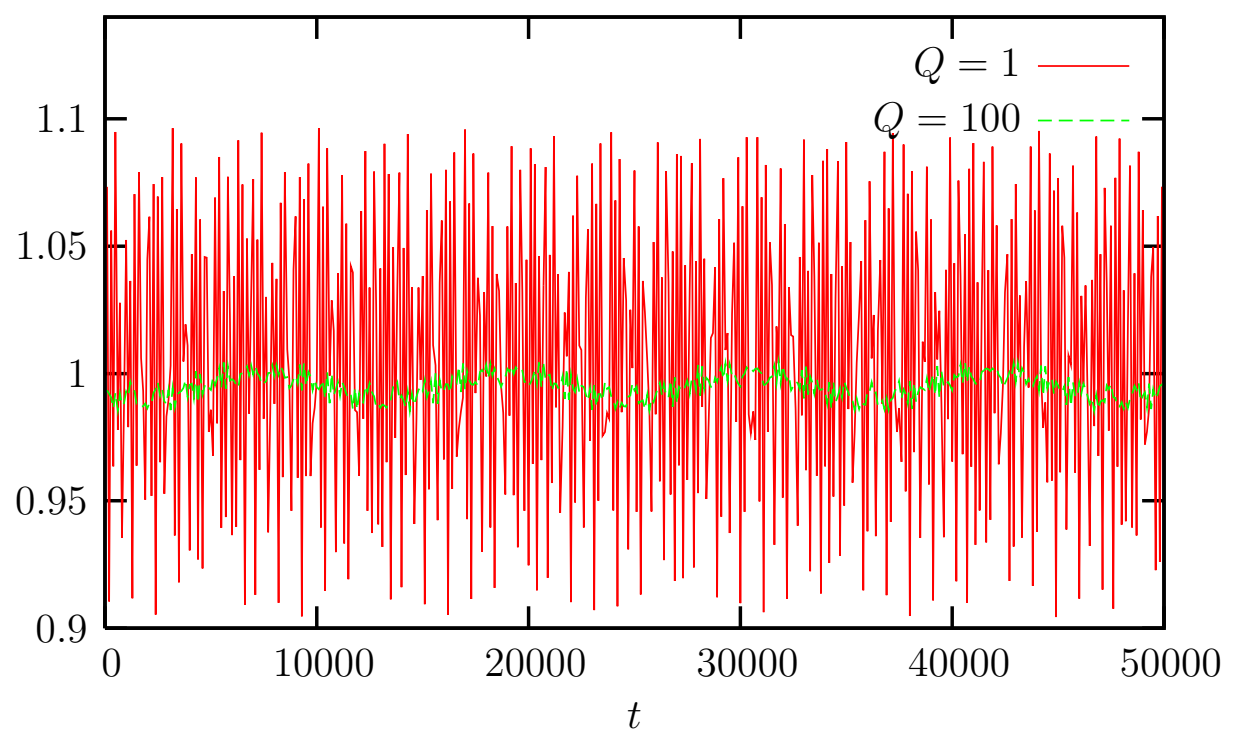

Figure 4. $E_{1}(q(t), p(t))$ (renormalized by its initial value) along the trajectory of (5), for $Q=1$ and $Q=100(\beta=1$, initial condition $q=(0 ; 0.5), p=(-1.5 ; 1.5), \xi=0)$.

We finally consider an initial condition such that $L\left(q_{0}, p_{0}\right)=0$. Along the trajectory of (5), we have $L(q(t), p(t))=0$ by (25), hence $E_{1}$ is not defined. On Figure 5, we plot

$$
E_{2}(q, p, \xi)=E_{2}\left(\tau(H(q, p)), \frac{\xi}{\sqrt{Q}}\right)
$$

along two trajectories, obtained with the same initial condition and the choices $Q=100$ and $Q=1$. We again observe that $E_{2}$ is almost constant for $Q=100$, and that it remains close to its initial value for $Q=1$.

On Figure 6, we plot the energy $H(q(t), p(t))$ along the same trajectory (for $Q=1$ ). We see that values $h \leq 1$ are not sampled. However, there exist $(q, p) \in \mathbb{R}^{4}$ such that $L(q, p)=0$ and $H(q, p)$ is as close to 0 as wanted. Hence, the trajectory only samples a strict subset of the level set $\{(q, p) ; L(q, p)=0\}$.

\section{Systems with one degree of freedom}

Consider a Hamiltonian system of the form (3) with $N=1$ and $M(q)=1$. All such systems are completely integrable since $H$ itself provides the required integral of motion. Suppose there is an interval of energies $I=\left[h_{1}, h_{2}\right]$ such that the level curves $M(h)=\{(q, p): H(q, p)=h\}, h \in I$, are all simple closed curves (one-dimensional 


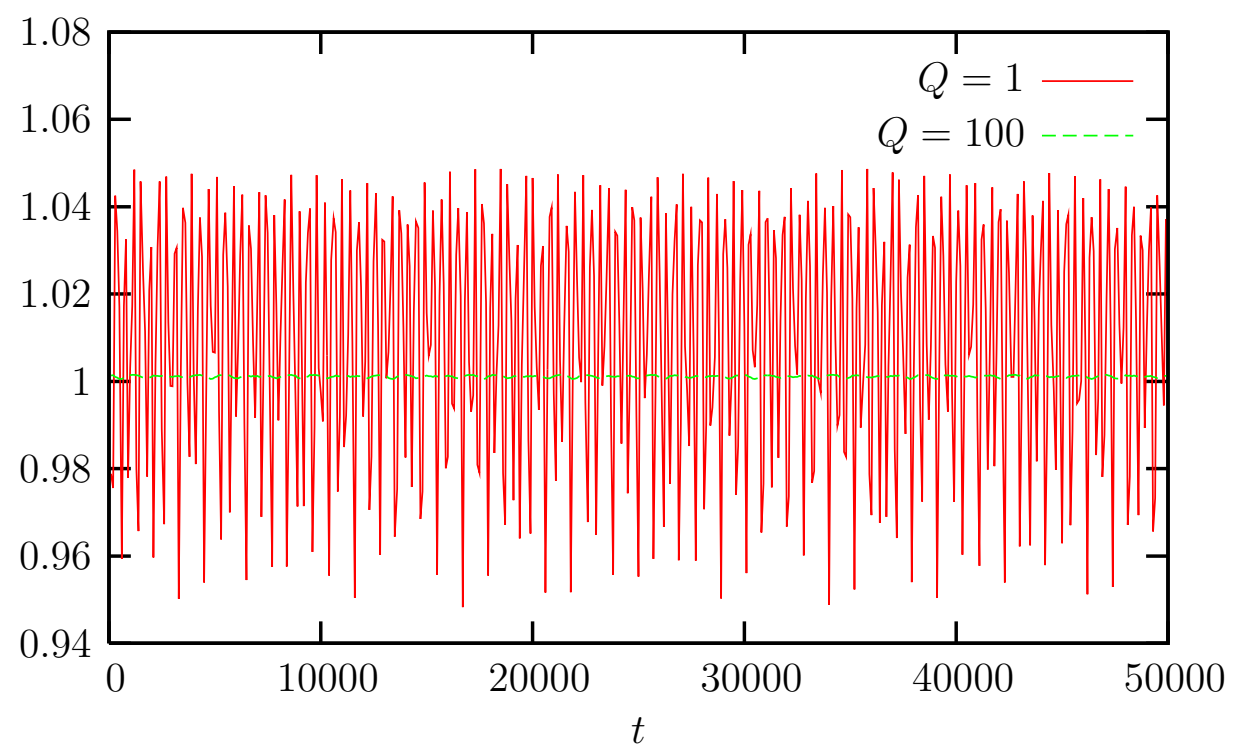

Figure 5. $E_{2}(q(t), p(t), \xi(t))$ (renormalized by its initial value) along the trajectory of (5), for $Q=1$ and $Q=100(\beta=1$, initial condition $q=(-0.5 ; 0.5), p=(-1 ; 1)$, $\xi=0)$.

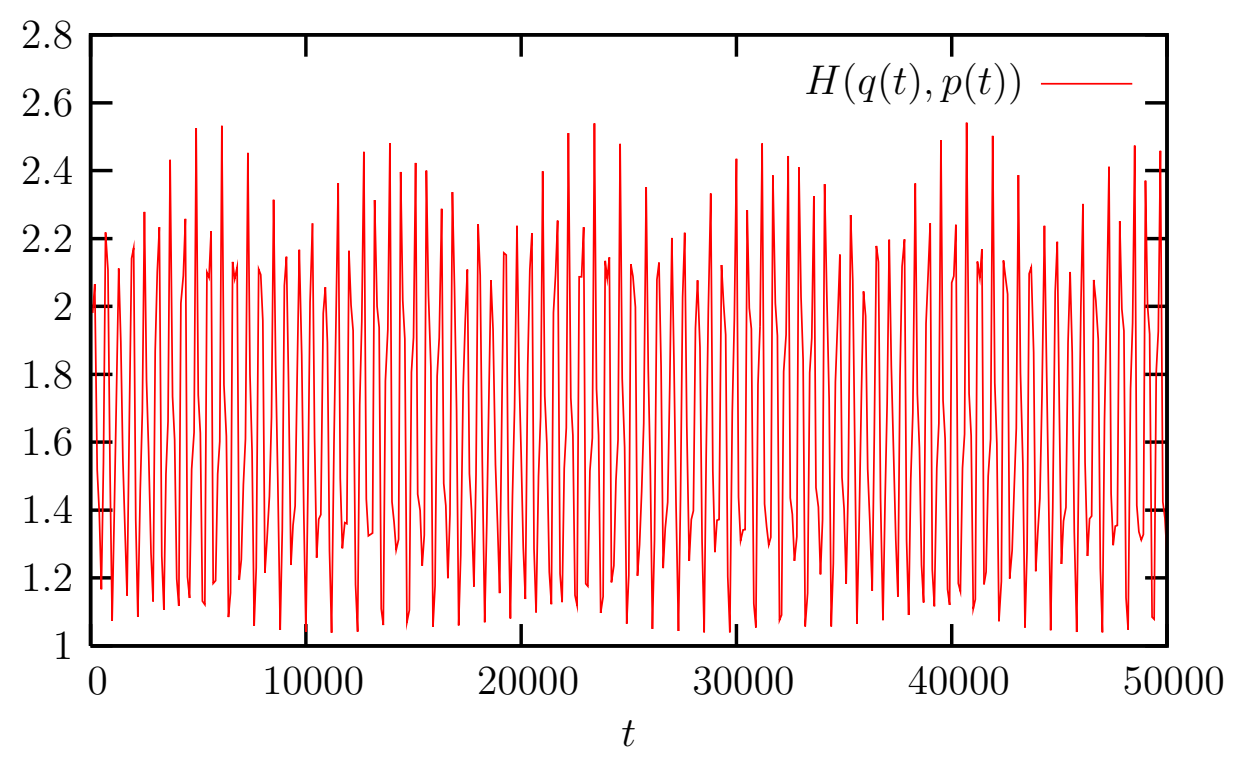

Figure 6. $H(q(t), p(t))$ along the trajectory of (5), for $Q=1(\beta=1$, initial condition $q=(-0.5 ; 0.5), p=(-1 ; 1), \xi=0)$.

tori) which are non-degenerate in the sense that the gradient of $H$ does not vanish. Then the plane region $U=\left\{(q, p): h_{1} \leq H(p, q) \leq h_{2}\right\}$ is diffeomorphic to an annulus, and we can introduce action-angle variables $(a, \theta)$ in $U$, and an exact symplectic map $\phi(\theta, a)=(q, p)$, as in Section 3.2.

From Theorem [3.2, we have $N=1$ first integrals for the averaged Nosé-Hoover 
equations. Let us now rewrite this first integral more explicitly. In view of (22), we have

$$
G(a, \alpha)=\frac{\alpha^{2}}{2}+W(a)
$$

with

$$
W(a)=\int^{a} \frac{k(s)}{s} d s,
$$

where, in view of (16), $k$ is given by

$$
k(a)=\int_{\mathbb{T}} \phi_{2}^{2}(\theta, a) d \theta-\frac{1}{\beta} .
$$

As in the multidimensional case, this integral prevents rapid ergodization, at least for small $\varepsilon$. But in the one-degree of freedom case we go further and identify conditions on $H$ which rigorously imply non-ergodicity. The method is essentially the one used in [9] where we treated the harmonic oscillator. Namely, the integral $G$ leads to invariant tori of the averaged system which, under certain assumptions, persist for small values of $\varepsilon$.

\subsection{Proof of non-ergodicity}

We will apply a KAM theorem to the Nosé-Hoover equations, in the formulation (14). Let us introduce the Poincaré return map, $P_{\varepsilon}$, of the system (14) to the Poincaré section defined by $\theta=0 \bmod 1$. It is convenient to rescale time by $\omega(a)$, so that the return time when $\varepsilon=0$ is 1 . This just alters the parametrization of the solutions so that the return time to the Poincare section is 1 .

Since there is only one degree of freedom, the averaging method can be rigorously justified. Indeed we can eliminate the fast angle $\theta$ of (14) by a change of variables. We construct functions $g(\hat{a}, \theta, \hat{\alpha})$ and $h(\hat{a}, \theta, \hat{\alpha})$ and corresponding new variables $(\hat{a}, \hat{\alpha})$ defined by

$$
\begin{aligned}
a & =\hat{a}+\varepsilon g(\hat{a}, \theta, \hat{\alpha}), \\
\alpha & =\hat{\alpha}+\varepsilon h(\hat{a}, \theta, \hat{\alpha}),
\end{aligned}
$$

so that in the new variables $(\hat{a}, \hat{\alpha})$, the dynamics (14) is given (after replacing $(\hat{a}, \hat{\alpha})$ by $(a, \alpha))$ by

$$
\begin{aligned}
\dot{\theta} & =\omega(a)+O(\varepsilon), \\
\dot{a} & =-\varepsilon \alpha S(a)+O\left(\varepsilon^{2}\right), \\
\dot{\alpha} & =\varepsilon k(a)+O\left(\varepsilon^{2}\right),
\end{aligned}
$$

where $S(a)$ and $k(a)$ are the averages (16).

In view of (35) and (36), the Poincaré map $P_{\varepsilon}(\hat{a}, \hat{\alpha})$ is an $O\left(\varepsilon^{2}\right)$ perturbation of the time $\varepsilon$ advance map of the averaged system (15), for which $G$ defined by (32) is a first integral. So we now make some assumptions about the level curves of $G$. 
Recall that we are working in a region $U$ of the $(q, p)$-plane defined by an interval of energies $I$ which corresponds to an interval of actions $J=\left[a_{1}, a_{2}\right]$. We assume that $W(a)$ has at least one local minimizer $a_{0}$ in $J$. We have

$$
0=W^{\prime}\left(a_{0}\right)=\frac{k\left(a_{0}\right)}{a_{0}}
$$

hence $k\left(a_{0}\right)=0$ and the point $P=\left(a_{0}, 0\right)$ is an equilibrium point for (15). The parts of the level curves of $G$ which are near $P$ are simple closed curves around $P$ in the $(a, \alpha)$-plane.

Remark 5.1 If $a_{0}$ is a local minimizer of $W$, then $k\left(a_{0}\right)=0$, hence $\int_{\mathbb{T}} \phi_{2}^{2}\left(\theta, a_{0}\right) d \theta=$ $\beta^{-1}$.

Let $G_{0}=G\left(a_{0}, 0\right)=W\left(a_{0}\right)$ be the value of the integral $G$ at the equilibrium point $P=\left(a_{0}, 0\right)$. Choose constants $\tilde{G}_{1}$ and $\tilde{G}_{2}$ such that $G_{0}<\tilde{G}_{1}<\tilde{G}_{2}<$ $\min \left(G\left(a_{1}, 0\right), G\left(a_{2}, 0\right)\right)=\min \left(W\left(a_{1}\right), W\left(a_{2}\right)\right)$ and let $\tilde{K}=\left[\tilde{G}_{1}, \tilde{G}_{2}\right]$ (see Figure 7). Then the level curves $\{(a, \alpha) ; G(a, \alpha)=c\}$, where $c \in \tilde{K}$, have connected components which are simple closed curves near $P$. The union of these components for $c \in \tilde{K}$ forms a region $\tilde{D}$ near $P$ which is diffeomorphic to an annulus.

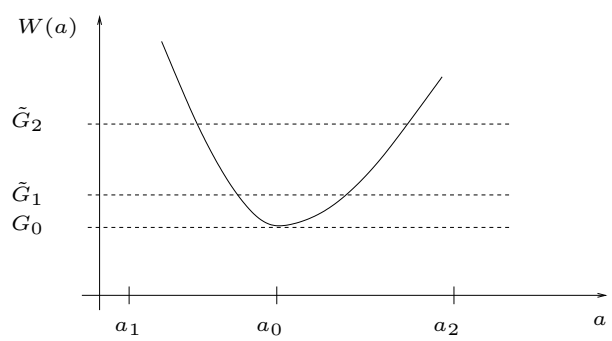

Figure 7. Schematic representation of the interval $\tilde{K}=\left[\tilde{G}_{1}, \tilde{G}_{2}\right]$.

The variable $G$ defines a natural action variable in $\tilde{D}$. We construct the corresponding angle variable $\phi$ by following the same method as in [9]. Let $T_{1}(g)$ denote the period of the periodic solutions of (15) which corresponds to the level curve $G=g \in \tilde{K}$. The averaged differential equation (15) becomes

$$
\begin{aligned}
\dot{\phi} & =1 / T_{1}(G), \\
\dot{G} & =0 .
\end{aligned}
$$

In these coordinates, the time $\varepsilon$ advance map takes the form $(\phi, G) \mapsto\left(\phi_{1}, G_{1}\right)$ where

$$
\begin{aligned}
\phi_{1} & =\phi+\varepsilon / T_{1}(G), \\
G_{1} & =G .
\end{aligned}
$$

Call this map $Q_{\varepsilon}(\phi, G)$. Then $P_{\varepsilon}(\phi, G)=Q_{\varepsilon}(\phi, G)+O\left(\varepsilon^{2}\right)$.

Theorem 5.1 Suppose the period function $T_{1}(G)$ is not identically constant on the interval $\tilde{K}$. Then, for $\varepsilon$ sufficiently small, the Poincaré map $P_{\varepsilon}$ has invariant circles in the region $\tilde{D}$ and so the Nosé-Hoover system is not ergodic: trajectories $(q(t), p(t))$ that solve (5) are not ergodic with respect to the Gibbs measure (2). 
Proof: As in [9], we apply Moser's twist theorem to the Poincaré map $P_{\varepsilon}$. The details are similar to those in [9], so we only sketch the argument here.

The fact that the Nosé-Hoover differential equation preserves the invariant measure (6) implies (as in [9]) that $P_{\varepsilon}$ preserves an invariant measure in the $(a, \alpha)$ plane. It follows that the maps $P_{\varepsilon}$ have the curve intersection property. The hypothesis on $T_{1}(G)$ guarantees that, making $\tilde{K}$ and hence $\tilde{D}$ smaller if necessary, we may assume that either $T_{1}^{\prime}(G)>0$ or $T_{1}^{\prime}(G)<0$ throughout $\tilde{D}$. This means that there are many invariant circles in $\tilde{D}$ for which the rotation number under $Q_{\varepsilon}$ is Diophantine. Moreover, the required twist condition holds. Moser's theorem guarantees that such invariant circles perturb to nearby invariant circles for $\varepsilon$ sufficiently small.

We now show that the existence of these invariant circles implies non-ergodicity with respect to the Gibbs measure. First, note that on a level curve $\mathcal{M}=$ $\{(a, \alpha) ; G(a, \alpha)=c\}$, where $c \in \tilde{K}$, we have that $W(a)$ is bounded from above, since $W(a) \leq G(a, \alpha)=c$. In view of the choice of $\tilde{K}$ (see Figure 7), this implies that $a$ is lower and upper bounded. Since $a^{\prime}(h)$ is positive and bounded away from 0 for $h \in I=\left[h_{1}, h_{2}\right]$, this hence shows that $H(q, p)$ is lower and upper bounded (that is, $|H(q, p)|$ is bounded) on the invariant circle $\mathcal{M}$ of $Q_{\varepsilon}$. As a consequence, $|H(q, p)|$ is bounded on the nearby invariant circles of $P_{\varepsilon}$. Hence, the trajectory of (5) does not sample values of $H(q, p)$ larger than some threshold. This is a contradiction with $(q(t), p(t))$ that solves (5) being ergodic with respect to the Gibbs measure (2).

Because of the complicated series of coordinate changes leading from the original Hamiltonian system to the averaged system, it is not easy to state simple conditions on the original potential function $V(q)$ which guarantee that the period function $T_{1}(G)$ is not constant. An equilibrium point surrounded by periodic orbits of constant period is called isochronous and various criteria for isochronicity have been given. Our problem can be reduced to a Hamiltonian case for which a simple criterion can be stated.

To carry out the reduction, replace $a$ in (15) (that is, (21) ) by $\sigma=\ln \left(a / a_{0}\right)$, where $a_{0}$ is a local minimizer of $W$ (see Figure 7). The differential equation (21) becomes

$$
\dot{\sigma}=-\alpha, \quad \dot{\alpha}=U^{\prime}(\sigma),
$$

where $U(\sigma)=W\left(a_{0} \exp \sigma\right)$. Except for a reversal of time, this is a classical Hamiltonian system with Hamiltonian $G(\sigma, \alpha)=\alpha^{2} / 2+U(\sigma)$. It has an equilibrium point at the origin $(\sigma, \alpha)=(0,0)$.

Now [8] discusses the problem of recovering the potential of such a system from its period function (see also [5]). Let $G_{0}=U(0)$ be the energy level of the equilibrium point at the origin. For $G>G_{0}$ let $L(G)$ be the width of the potential well at energy $G$, i.e., $L(G)=\sigma_{2}(G)-\sigma_{1}(G)$ where $\sigma_{i}(G)$ are the two roots of $U(\sigma)=G$ near $\sigma=0$. Then $T_{1}(G)$ is constant if and only if $L(G)=\frac{T_{1}}{\pi} \sqrt{2\left(G-G_{0}\right)}$. This is just the formula for the width of the quadratic potential well associated to a harmonic oscillator of period $T_{1}$. Clearly this is highly exceptional and is easy to rule out, at least numerically.

Another way to show that $T_{1}(G)$ is non-constant is to observe that the constancy of the period implies that the family of periodic orbits surrounding the equilibrium 
point must fill the entire plane [5]. If this were not so, then there would be another equilibrium point on the boundary of the maximal family which would force $T_{1}(G) \rightarrow \infty$. For example, for certain values of $\beta$, the pendulum equations (see Section 5.2) lead to an averaged system with more than one equilibrium, and this immediately implies that $T_{1}(G)$ is non-constant.

\subsection{The simple pendulum problem}

We consider here the numerical example of a simple pendulum whose potential energy is given by

$$
V(q)=-\cos q
$$

We reduce $q$ modulo $2 \pi$. By construction, the energy satisfies $h \geq-1$. The phase portrait is shown on Figure 8. The above assumptions are satisfied for energies in the interval $I=\left[h_{1}, h_{2}\right]$, with $-1<h_{1}<h_{2}<1$, or $1<h_{1}<h_{2}$.

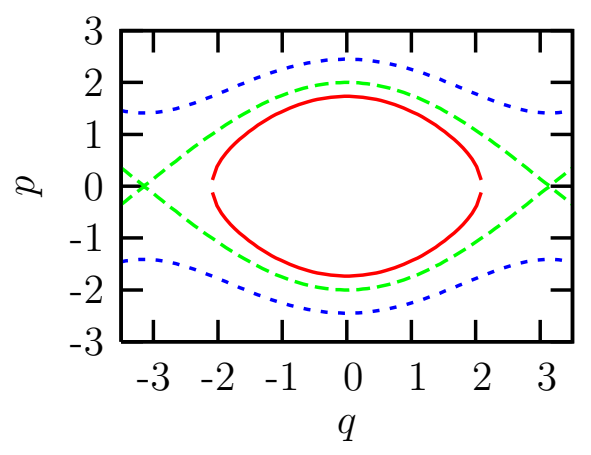

Figure 8. Phase portrait of the simple pendulum.

First, we numerically compute $a(h)$ defined by (19). Note that, in this onedimensional setting,

$$
a(h)=\int_{M(h)} p d q
$$

where the line integral is taken in the direction of the Hamiltonian flow, and $M(h)=$ $\left\{(q, p) \in \mathbb{R}^{2}: H(q, p)=h\right\}$. We also compute

$$
k_{0}(a)=\int_{\mathbb{T}} \phi_{2}^{2}(\theta, a) d \theta
$$

which is independent of $\beta$ and satisfies $k(a)=k_{0}(a)-\beta^{-1}$, with $k$ defined by (34). In practice, $k_{0}$ is computed using the fact that, for any energy level $h$,

$$
k_{0}(a(h))=\lim _{T \rightarrow+\infty} \frac{1}{T} \int_{0}^{T} p^{2}(t) d t,
$$

where $(q(t), p(t))$ solve the Newton equations of motion for the pendulum at the constant energy $h$. Results are shown on Figure 9. 

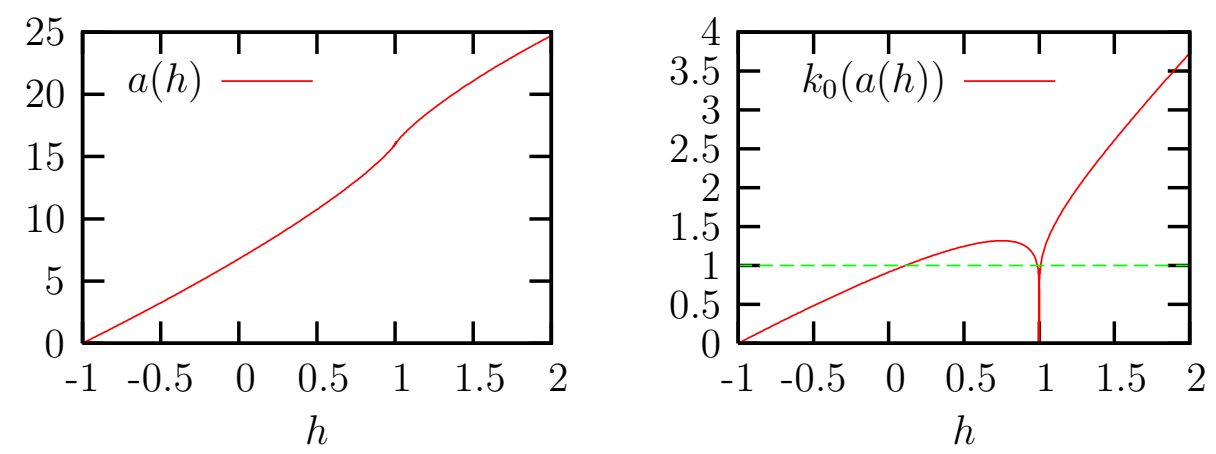

Figure 9. Numerically computed values of $a(h)$ and $k_{0}(a(h))$ (see text).

We have seen that the action values $a$ such that $k_{0}(a)=\beta^{-1}$, that is $k(a)=0$, play an important role (see Remark 5.1). In view of Figure 9, we see that, when $\beta^{-1}<\beta_{c}^{-1}$ for some threshold $\beta_{c}$, then the equation $k_{0}(a)=\beta^{-1}$ has three solutions. When $\beta^{-1}>\beta_{c}^{-1}$, then the equation $k_{0}(a)=\beta^{-1}$ has a unique solution. In what follows, we detail the numerical results obtained with the choice $\beta=1$, which corresponds to the first case. Similar results have been obtained for choices of $\beta$ corresponding to the other case. Hence, the conclusions that we draw here are by no means restricted to the case $\beta=1$.

The function $W(a)$ defined by (33) is shown on Figure 10 for the choice $\beta=1$. This function has two local minimizers, $a_{1} \approx 7.6$ and $a_{2} \approx 16.17$. Following the above theoretical analysis, we work close to one of them. We have chosen to work close to $a_{1}$.

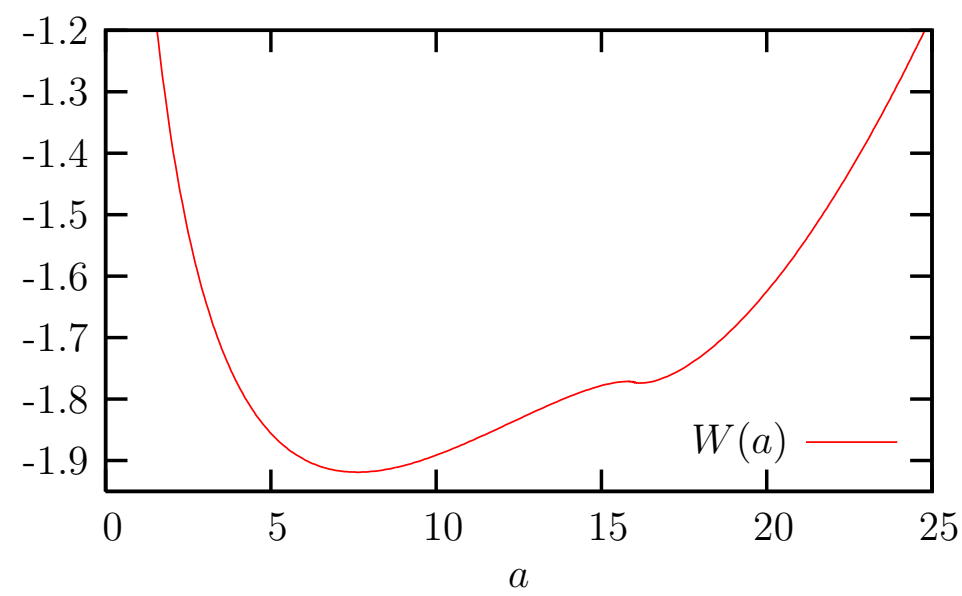

Figure 10. Numerically computed values of $W(a)$ for $\beta=1$.

On Figure 11, we plot the trajectory of the averaged dynamics (15) for different initial conditions. These trajectories have been computed with the Symplectic Euler algorithm used on the Hamiltonian formulation (39). As expected, the trajectory is a simple closed curve around the equilibrium point $\left(a_{1}, 0\right)$, that corresponds to a level curve of $G$. These curves are also invariant curves of the map $Q_{\varepsilon}$ defined in the previous section (see map (38)). 


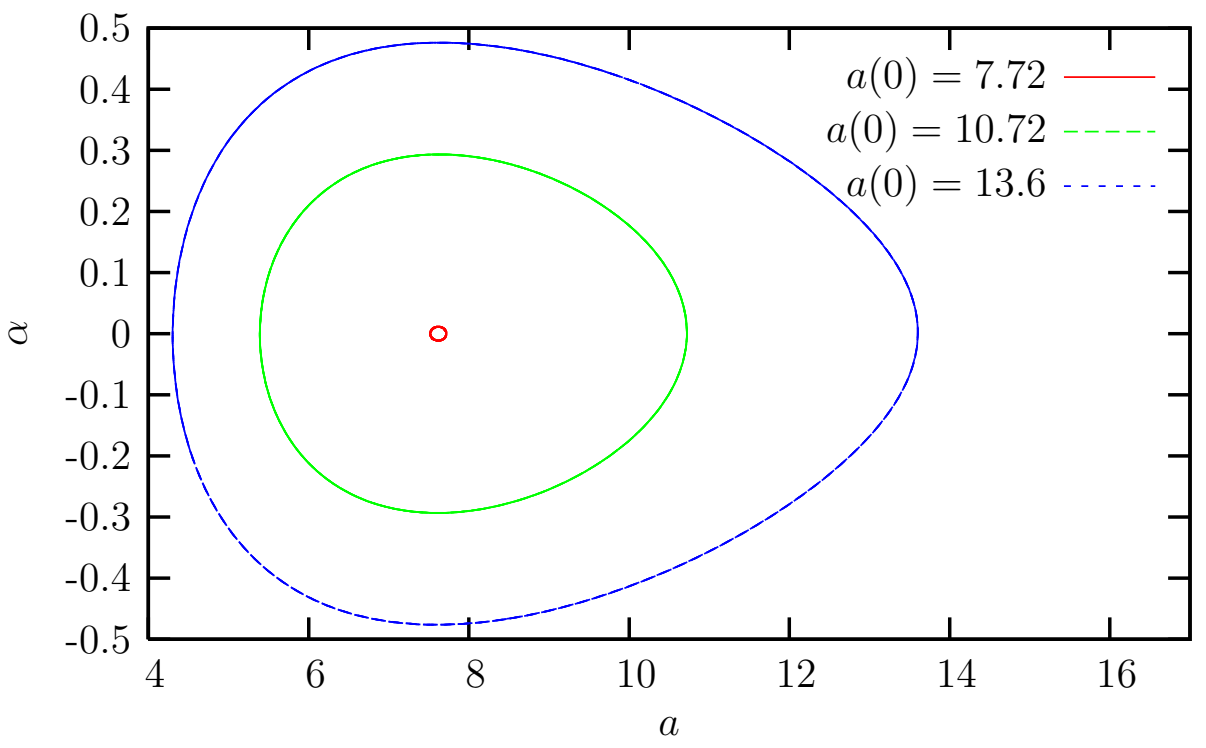

Figure 11. Trajectories of (15) for several different values of $a(0)(\beta=1)$.

We now study how these curves persist upon perturbation. We recall that the Poincaré return map $P_{\varepsilon}$ of the Nosé-Hoover dynamics (14) on the section $\theta=0 \bmod 1$ is a perturbation of $Q_{\varepsilon}$. Results for the Poincaré return map of the dynamics (14) are shown on Figure 12 for $Q=10^{5}$ (that is, $\varepsilon=\sqrt{10} \times 10^{-3}$ ), and on Figure 13 for $Q=1$ (that is, $\varepsilon=1$ ), for the same initial energies as for Figure 11. These Poincaré return maps have been computed using the fact that the section $\theta=0 \bmod 1$ corresponds to the section $q=0 \bmod 2 \pi$. We see a good agreement between Figures 11$]$ and 12, The presence of invariant circles on Figures 12 and 13 shows that the system (14) seems to have invariant curves, for $Q=10^{5}$ and $Q=1$.

Note that Theorem 5.1, which states the non-ergodicity of the Nosé-Hoover equations, relies on the important assumption that the period $T_{1}(G)$ of the averaged equations is not constant. This holds true for the pendulum case, in view of the discussion at the end of Section 5.1. This is also confirmed by numerical computations of $T_{1}(G)$ (see Figure 14).

Let us now look at another criterion for ergodicity, namely what energy values are sampled. We see on Figure 13 that small values of $a$ are not sampled: we have $a \geq 6$ for the three initial conditions that we considered. In view of Figure 9, this corresponds to small values of $H$ not being sampled. On Figure 15, we plot the physical energy $H(q(t), p(t))$ along the trajectory of (14), for the value $Q=1$, and the initial condition $q=0, p=1.5, \xi=0$, that corresponds to the initial value $a(0)=7.72$ that we studied on Figures 11, 12 and 13 (results are the same for other initial conditions). We see that $H \geq-0.4$. If the dynamics (14) was sampling the canonical measure, then all values of $H$ would be attained. In particular, the smallest values $H \approx-1$ would be the most frequent ones. Indeed, from the Gibbs measure (21), we compute the probability distribution function of the energy, which reads $\rho(h)=z^{-1} \exp (-\beta h) a^{\prime}(h)$, where $z$ 




Figure 12. Poincaré return map of (14) on the plane $\theta=0 \bmod 1$ for several initial conditions $\left(Q=10^{5}, \beta=1\right)$.

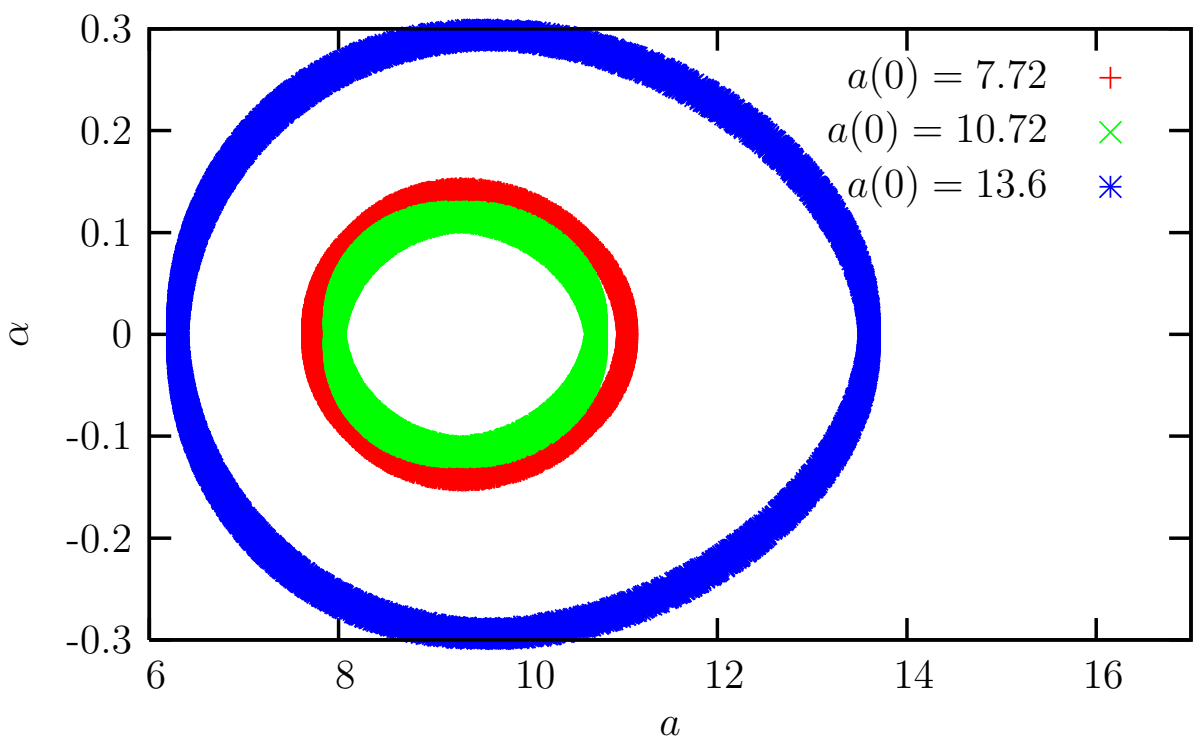

Figure 13. Poincaré return map of (14) on the plane $\theta=0 \bmod 1$ for several initial conditions $(Q=1, \beta=1)$.

is a normalization constant. For the pendulum case, $a^{\prime}(h)$ is close to a constant (see Figure 91), hence the smallest values of $h$ are the most frequent ones. Hence, it seems that (14) is not ergodic with respect to the canonical measure, even for the value $Q=1$.

\section{Acknowledgments}

Part of this work was completed while the first author was visiting the Institute for Mathematics and its Applications (Minneapolis), whose hospitality is gratefully 


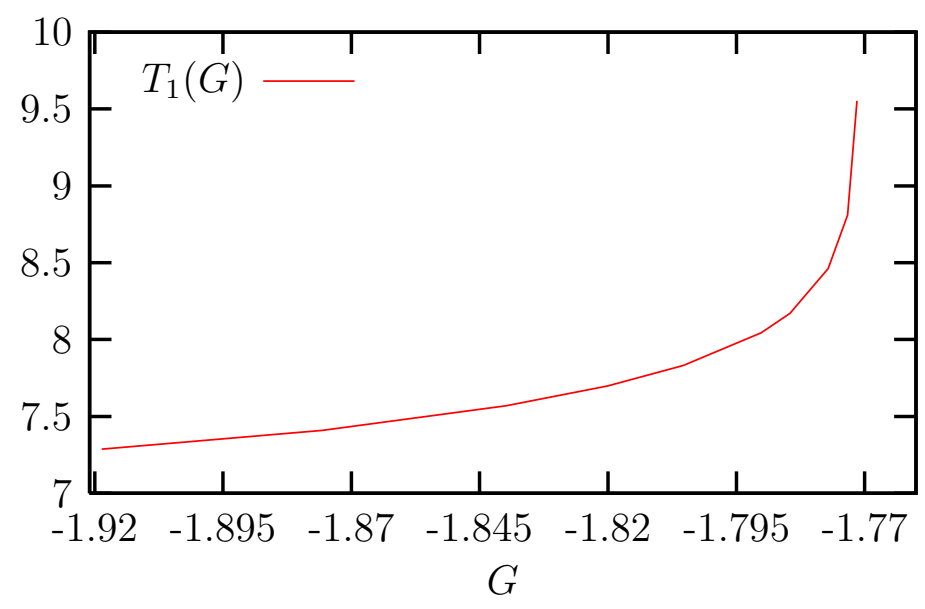

Figure 14. Period $T_{1}(G)$ of the averaged equation (15) $(\beta=1)$.

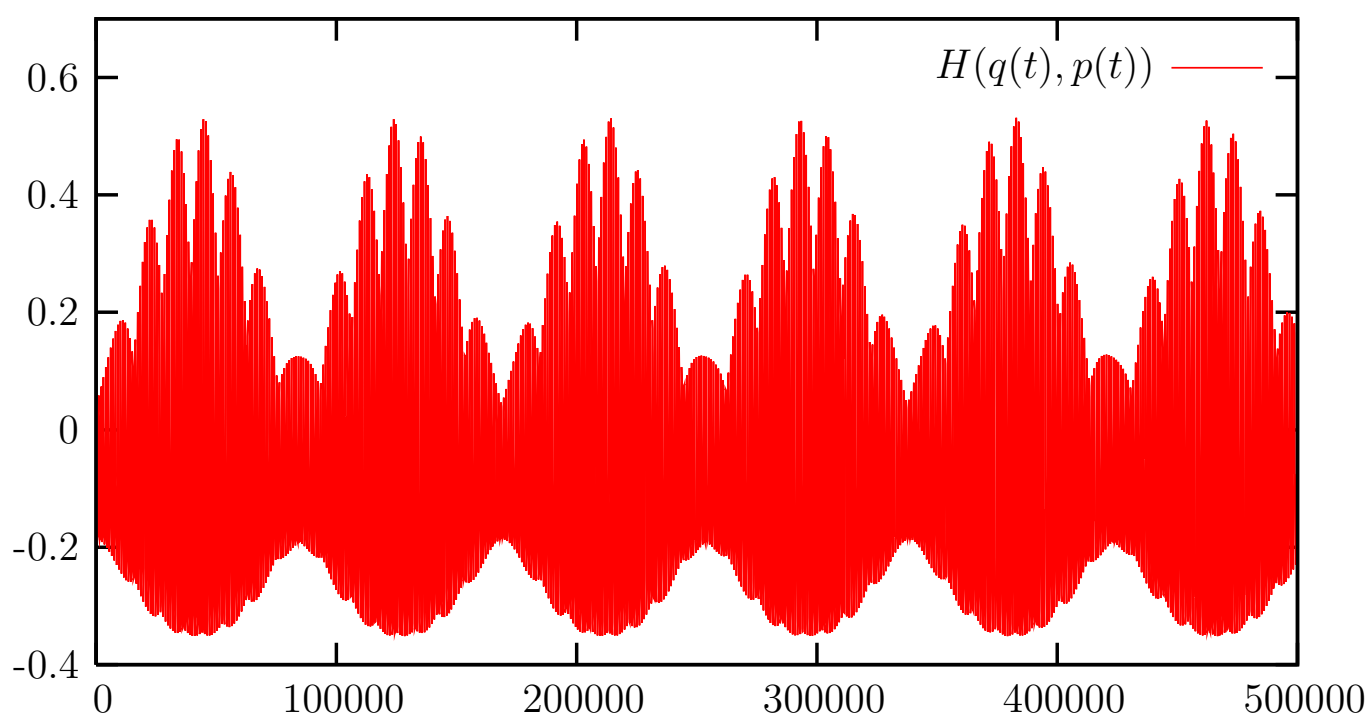

Figure 15. Energy $H(q(t), p(t))$ along the trajectory of (5), for $Q=1$ and $\beta=1$, and the initial condition $q=0, p=1.5, \xi=0$ (that is, $a(0)=7.72$ ).

acknowledged.

The work of Frédéric Legoll was supported in part by the Agence Nationale de la Recherche (INGEMOL non-thematic program) and by the Action Concertée Incitative "Nouvelles Interfaces des Mathématiques" SIMUMOL (Ministère de la Recherche et des Nouvelles Technologies, France).

The work of Mitchell Luskin was supported in part by NSF Grants DMS-0757355 and DMS-0811039, the Institute for Mathematics and its Applications, and by the University of Minnesota Supercomputing Institute. This work is also based on work supported by the Department of Energy under Award Number DE-FG02-05ER25706.

Richard Moeckel was partially supported by NSF Grant DMS-0500443. 


\section{References}

[1] V.I. Arnold. Mathematical Methods of Classical Mechanics. Springer Verlag, New York, 1989.

[2] V.I. Arnold, V.V. Kozlov, and A.I. Neishtadt. Mathematical Aspects of Classical and Celestial Mechanics. Springer, 2nd ed., 1997.

[3] S.D. Bond, B.J. Leimkuhler, and B.B. Laird. The Nosé-Poincaré method for constant temperature molecular dynamics. J. Comput. Phys., 151:114-134, 1999.

[4] E. Cancès, F. Legoll, and G. Stoltz. Theoretical and numerical comparison of some sampling methods for molecular dynamics. Math. Mod. Num. Anal. (M2AN), 41(2):351-389, 2007.

[5] A. Cima, F. Manosas, and J. Villadelprat. Isochronicity for several classes of Hamiltonian systems. Jour. Diff. Eq., 157:373-413, 1999.

[6] D. Frenkel and B. Smit. Understanding Molecular Simulation, from algorithms to applications, 2nd ed. Academic Press, 2002.

[7] W. Hoover. Canonical dynamics: Equilibrium phase-space distributions. Phys. Rev. A, 31(3):1695-1697, 1985.

[8] L.D. Landau and E.M. Lifshitz. Mechanics. Pergamon Press, Oxford, 2nd ed., 1969.

[9] F. Legoll, M. Luskin, and R. Moeckel. Non-ergodicity of the Nosé-Hoover thermostatted harmonic oscillator. Arch. Rat. Mech. Anal., 184(3):449-463, 2007.

[10] B.J. Leimkuhler, E. Noorizadeh, and F. Theil. private communication.

[11] B.J. Leimkuhler and C.R. Sweet. A Hamiltonian formulation for recursive multiple thermostats in a common timescale. SIAM J. App. Dyn. Sys., 4(1):187-216, 2005.

[12] G. Martyna, M. Klein, and M. Tuckerman. Nosé-Hoover chains: The canonical ensemble via continuous dynamics. J. Chem. Phys., 97(4):2635-2643, 1992.

[13] G. Martyna, M. Tuckerman, D. Tobias, and M. Klein. Explicit reversible integrators for extended systems dynamics. Mol. Phys., 87:1117-1157, 1996.

[14] D. McQuarrie. Statistical Mechanics. University Science Books, 2000.

[15] S. Nosé. A unified formulation of the constant temperature molecular dynamics method. J. Chem. Phys., 81(1):511-519, 1984.

[16] M. Tuckerman and G. Martyna. Understanding modern molecular dynamics: Techniques and applications. J. Phys. Chem. B, 104:159-178, 2000. 